\title{
Structure of Melaminium Dihydrogenpyrophosphate and Its Formation from Melaminium Dihydrogenphosphate Studied with Powder Diffraction Data, Solid-State NMR, and Theoretical Calculations
}

\author{
Vladimir Brodski, ${ }^{*}, \dagger$ René Peschar, ${ }^{\dagger}$ Henk Schenk, ${ }^{\dagger}$ Andreas Brinkmann,,$\dagger$ \\ Ernst R. H. van Eck, ${ }^{*}$ Arno P. M. Kentgens, ${ }^{\ddagger}$ Betty Coussens, $^{\S}$ and Ad Braam ${ }^{\S}$ \\ Universiteit van Amsterdam, van't Hoff Institute for Molecular Sciences, Laboratory for Crystallography, \\ Nieuwe Achtergracht 166, NL-1018WV Amsterdam, The Netherlands, University of Nijmegen, \\ Physical Chemistry/Solid State NMR, NSRIM Center, Toernooiveld 1, NL-6525 ED Nijmegen, The Netherlands, \\ and DSM Research, Postbus 18, 6160 MD, Geleen, The Netherlands
}

Received: June 2, 2004; In Final Form: July 23, 2004

\begin{abstract}
Melaminium dihydrogenpyrophosphate (bis(2,4,6-triamino-1,3,5-triazinium dihydrogenpyrophosphate, MPy), obtained as a crystalline powder by thermal treatment of melaminium dihydrogenphosphate (MP), is the first intermediate in the synthesis of polymerized melamine phosphates that are used as environmental-friendly flame retardants. The crystal structure of MPy was determined by X-ray powder diffraction and the proposed hydrogen-bonding model was corroborated by solid-state NMR and periodic DFT calculations. A mechanism is proposed for the endothermic dehydration process that takes place in the reaction from MP to MPy, in accordance with energy calculations based on the optimized crystal structures of MPy and MP. A packing analysis of MPy and other melamine-containing compounds revealed some typical melamine packing motifs.
\end{abstract}

\section{Introduction}

Melamine salts of phosphoric acid are widely used as additives in polymers to improve the latters flame retardancy characteristics. $^{1-5}$ In this respect, melamine phosphates are considered as promising replacements of halogenated fire retardants, which show undesirable side effects such as toxic and corrosive smoke when being burnt. ${ }^{6}$

Large-scale use of melamine phosphates has been hampered by a lack of fundamental knowledge of their structural characteristics ${ }^{1}$ as until recently only one compound was solved from single-crystal data ${ }^{7}$ and most melamine phosphates ${ }^{2,8}$ are available only as microcrystalline powders. In recent years, however, considerable progress has been made with the determination of structures of molecular crystals directly from powder diffraction data. ${ }^{9}$ In addition solid-state NMR has proven to be well-suited to investigate hydrogen-bonded systems such as benzoxazine dimers and oligomers. ${ }^{10-13}$ Earlier, we have successfully employed a combined approach of powder diffraction and solidstate NMR to determine the crystal structure of melaminium dihydrogenphosphate $\mathrm{C}_{3} \mathrm{H}_{7} \mathrm{~N}_{6}{ }^{+} \cdot \mathrm{H}_{2} \mathrm{PO}_{4}^{-}$(from now on abbreviated as melamine phosphate; MP). ${ }^{14,15}$ In a continuation of this work, we report here the structure of melaminium dihydrogenpyrophosphate $\left(\mathrm{C}_{3} \mathrm{H}_{7} \mathrm{~N}_{6}{ }^{+}\right)_{2} \cdot \mathrm{H}_{2} \mathrm{P}_{2} \mathrm{O}_{7}{ }^{2-}$ (from now on abbreviated as melamine pyrophosphate; MPy) that is formed when heating MP. On the basis of the crystal structure models of MP and MPy the dehydration process that is important for understanding the FR's activity ${ }^{2}$ will be discussed.

Based on an analysis of all melamine-containing compounds known to date, the melamine-packing motifs in such compounds

* To whom correspondence should be addressed. E-mail: brodski@ science.uva.nl

$\dagger$ Universiteit van Amsterdam.

$\doteqdot$ University of Nijmegen.

$\S$ DSM Research. will be discussed. These packing characteristics are expected to be important for future structure determination of melaminecontaining compounds.

\section{Experimental Section}

Preparation of MPy. MPy (white crystalline powder) was synthesized by heating MP at $240{ }^{\circ} \mathrm{C}$ for at least $2 \mathrm{~h}$.

X-ray Diffraction. An X-ray powder diffraction pattern of MPy was collected at room temperature with an Enraf-Nonius FR 552 Guinier Johansson camera equipped with a Johansson monochromator ${ }^{16}$ and with $\mathrm{Cu} \mathrm{K} \alpha_{1}$ radiation $(\lambda=1.54060 \AA)$. The sample was prepared by pressing the powder into a thin layer onto Mylar foil and during data collection the sample was rotated to improve particle statistics. For the structure determination and initial Rietveld refinement, the Guinier photograph was read out with an optical instrument and, using a Johansson LS-18 microdensimeter, digitized to a pattern with a step size of $0.01^{\circ} 2 \theta$.

For the stage of the Rietveld refinement, a high-resolution $\mathrm{X}$-ray powder diffraction pattern was collected at beamline BM01B of the Swiss-Norwegian CRG at the European Synchrotron Radiation Facility (ESRF, Grenoble) with a fixed wavelength of $0.79942 \AA$ at room temperature. Data collection was carried out in a continuous scan mode from $1.0^{\circ}$ to $43.0^{\circ}$ $2 \theta$ using a filled capillary (diameter $1.5 \mathrm{~mm}$ ) that was rotated during exposure. After data collection the data were binned at $0.005^{\circ} 2 \theta$

Solid-State NMR Spectroscopy. Single-pulse ${ }^{1} \mathrm{H}$ MAS solidstate NMR spectra of MP and MPy were obtained at a static field of $18.8 \mathrm{~T}$ (Varian Unity) and sample spinning frequency of $49.0 \mathrm{kHz}$ on a home-built probehead based on a Samosontype $^{17}$ magic-angle-spinning (MAS) assembly. The ${ }^{31} \mathrm{P}$ crosspolarization magic-angle-spinning (CP-MAS) spectrum was acquired at a static field of $7.05 \mathrm{~T}$ (Chemagnetics Infinity) and 
a sample spinning frequency of $4.0 \mathrm{kHz}$ with a $\mathrm{CP}$ contact time of $4 \mathrm{~ms}$. TPPM decoupling ${ }^{18}$ with a ${ }^{1} \mathrm{H}$ nutation frequency of $100 \mathrm{kHz}$, pulse durations of $5.3 \mu \mathrm{s}$, and radio frequency phases of $\pm 40^{\circ}$ was used during acquisition. The two-dimensional ${ }^{1} \mathrm{H}-$

${ }^{31} \mathrm{P}$ heteronuclear correlation spectrum of MPy was obtained at a static field of $7.05 \mathrm{~T}$ and a sample spinning frequency of 12.0 kHz. Frequency-switched Lee-Goldburg homonuclear decoupling ${ }^{19}$ with a nutation frequency of $100 \mathrm{kHz}$, frequency jumps of $\pm 70.711 \mathrm{kHz}$, and an overall offset of $-1.5 \mathrm{kHz}$ was used in the indirect ${ }^{1} \mathrm{H}$ dimension. The ${ }^{1} \mathrm{H}$ magnetization was transferred to ${ }^{31} \mathrm{P}$ using Lee-Goldburg cross-polarization ${ }^{20}$ with a duration of $0.5 \mathrm{~ms}$. TPPM decoupling ${ }^{18}$ with a ${ }^{1} \mathrm{H}$ nutation frequency of $100 \mathrm{kHz}$, pulse durations of $5 \mu \mathrm{s}$, and radio frequency phases of $\pm 25^{\circ}$ was applied during acquisition.

Theoretical Calculations. To validate the hydrogen-bonding model proposed for the MPy structure, periodic DFT computations have been carried out with use of the CASTEP code. ${ }^{21}$ Keeping the unit cell parameters fixed at their experimental values, models with various hydrogen positions were optimized with the exchange correlation functional of Perdew, Berke, and Ernzerhof $(\mathrm{PBE})^{22}$ in combination with Vanderbilt ultra-soft pseudopotentials. ${ }^{23}$ So-called "medium" precision was employed implying an energy cutoff of $300 \mathrm{eV}$, an SCF tolerance of 2.0 $10^{-6} \mathrm{eV} /$ atom, a $k$-point separation of $0.05 \AA^{-1}$, and geometry convergence tolerances of $2.010^{-5} \mathrm{eV} /$ atom for the total energy, $0.05 \mathrm{eV} / \AA$ for the maximum force, $0.01 \mathrm{GPa}$ for the maximum stress, and $0.002 \AA$ for the maximum displacement.

To understand the energy balance of the condensation reaction of MP to MPy,

$$
2\left(\mathrm{C}_{3} \mathrm{H}_{7} \mathrm{~N}_{6}{ }^{+} \cdot \mathrm{H}_{2} \mathrm{PO}_{4}{ }^{-}\right) \stackrel{\Delta T}{\longrightarrow}\left(\mathrm{C}_{3} \mathrm{H}_{7} \mathrm{~N}_{6}{ }^{+}\right)_{2} \cdot \mathrm{H}_{2} \mathrm{P}_{2} \mathrm{O}_{7}{ }^{2-}+\mathrm{H}_{2} \mathrm{O}
$$

the energies of MP and MPy were calculated by performing optimizations as discussed above, using the respective crystal structures as starting points. The energy of the (free) water molecule was computed by optimizing a single water in a periodic box of $10 \times 10 \times 10 \AA^{3}$.

\section{Results}

Crystal Structure Determination of MPy from Powder X-ray Diffraction Data. The unit cell was determined with the indexing program $\mathrm{ITO}^{24}$ (Table 1). For indexing, the accurate positions of 48 lines in the interval $2 \theta=0.0-40.0^{\circ}$ were read off from the Guinier photograph. A number of weak lines could not be indexed, indicating a small amount $(1-2 \%)$ of another crystalline phase. In view of the cell volume, and assuming that MPy is crystallized in space group $P \overline{1}$ with $\mathrm{Z}=2$, the calculated density is $1.80 \mathrm{~g} \mathrm{~cm}^{-3}$. This value is in good agreement with the density $d_{\mathrm{m}}=1.81 \mathrm{~g} \mathrm{~cm}^{-3}$ as measured at DSM.

Structure solution was carried out with the Monte Carlo program Organa. ${ }^{25}$ An initial molecular model of MPy was built consisting of the melamine moiety from the crystal structure of $\mathrm{MP}^{14}$ while the pyrophosphate moiety $\left(\mathrm{P}_{2} \mathrm{O}_{7}{ }^{4-}\right)$ was taken from the crystal structure of guanidinium pyrophosphate monohydrate. ${ }^{26}$ Only non-hydrogen atoms were considered at this stage.

The model found by Organa $\left(R_{\mathrm{wp}} \approx 13 \%\right)$ was refined by using the Rietveld module of the program MRIA, ${ }^{27}$ using a splittype pseudo-Voigt peak profile function ${ }^{28}$ for peaks in the range $2 \theta=4.00-43.00^{\circ}$ and modeling the preferred orientation with the March-Dollase expression. ${ }^{29}$ To avoid distortion of the model, bond restraints as described by Baerlocher ${ }^{30}$ were applied to all interatomic distances up to $3.0 \AA$ (with $\sigma=1 \%$ of the ideal distance). The weight $c_{\mathrm{w}}$ (see ref $30, \mathrm{p} 188$ ) that weighs the residual function of the restraints $S_{R}$ versus the Rietveld
TABLE 1: Crystallographic Data of Melamine Pyrophosphate

$\begin{array}{ll}\text { formula } & \left(\mathrm{C}_{3} \mathrm{H}_{7} \mathrm{~N}_{6}{ }^{+}\right)_{2} \cdot \mathrm{H}_{2} \mathrm{P}_{2} \mathrm{O}_{7}{ }^{2-} \\ \text { crystal system } & \text { triclinic } \\ \text { space group } & P \overline{1}(\text { no. } 2) \\ Z & 2 \\ \text { lattice constants } & \\ a(\AA) & 10.89321(2) \\ b(\AA) & 11.20792(2) \\ c(\AA) & 6.84243(1) \\ \alpha(\mathrm{deg}) & 104.969(1) \\ \beta(\text { deg) } & 101.473(1) \\ \gamma(\mathrm{deg}) & 83.805(1) \\ V\left(\AA^{3}\right) & 789.50(2) \\ \left.d_{\mathrm{m}} / d_{\mathrm{c}}(\mathrm{gcm})^{-3}\right) & 1.81 / 1.80 \\ \text { data collection } & \\ \text { data range } & 4.00^{\circ} \leq 2 \theta \leq 43.00^{\circ} \\ \text { no. of data points } & 7800 \\ \text { no. of reflns } & 2220 \\ \text { temp }(\mathrm{K}) & 294 \\ \text { structure refinement } & \\ \text { GoF } & 6.71 \\ R_{\mathrm{p}}(\%) & 3.71 \\ R_{\text {wp }}(\%) & 4.97 \\ \text { no. of refined parameters } & \\ \text { lattice } & 6 \\ \text { positional } & 27 \\ \text { thermal } & 4 \\ \text { texture } & 14 \\ \text { profile } & 28 \\ \text { background } & 12 \\ \text { zero shift } & 1 \\ \end{array}$

residual $S_{Y}$ was reduced gradually (from the starting value of 691) and at the final stages the restraints were completely removed $\left(\mathrm{S}_{\mathrm{Y}}=0\right)$. $U_{\text {iso }}$ values of identical atom types were coupled and a small damping was applied during all refinement stages.

Apart from the hydrogens bonded to the exocyclic nitrogens, assumed to be in the usual planar $-\mathrm{NH}_{2}$ conformation, also four other hydrogens had to be positioned to neutralize the charge. An analysis of the short intermolecular contacts suggested four possible hydrogen bonds, comprising 16 different protonation models. Refinement of any of the hydrogens, even those bonded to the exocyclic nitrogens, led to unacceptable hydrogen positions. Therefore, all hydrogens had to be calculated and kept at a fixed distance from their carrier. One of the hydrogen models, consisting of protonated melamines and dihydrogen pyrophosphate (Figure 1), was chosen to be the most likely because the $\mathrm{H}-\mathrm{O}$ bonds did not increase dramatically during the refinement.

Some other arguments concerning the molecular structure of melamine and pyrophosphate moieties support the protonation model shown in Figure 1. It seems likely that hydrogen H34 is bonded to the endocyclic N5 instead of to $\mathrm{O} 23$ because the relatively short distance of the P19-O23 ( 1.47 $\AA$ ) bond suggests a double bond rather than a hydrogen-bonded oxygen. Protonation of the endocyclic nitrogen N14 (instead of O26) in the other melamine moiety, however, cannot be inferred on the basis of the same argumentation. Nevertheless, significant distortions of the melamines suggest that both rings are protonated. In contrast to, for example, crystalline melamine ${ }^{31}$ but similar as in MP, in MPy the aromatic $\pi$-system is distorted because of proton transfer from the (pyro)phosphates to one of the endocyclic melamine nitrogens. In particular, this leads to a bond length alteration with $\mathrm{C}-\mathrm{N}$ distances varying in the range 1.28-1.47 $\AA$. The internal $\mathrm{C}-\mathrm{N}-\mathrm{C}$ bond angles at the protonated endocyclic nitrogens are larger than the other two $\mathrm{C}-\mathrm{N}-\mathrm{C}$ angles of the rings by about $3-6^{\circ}$. These differences 


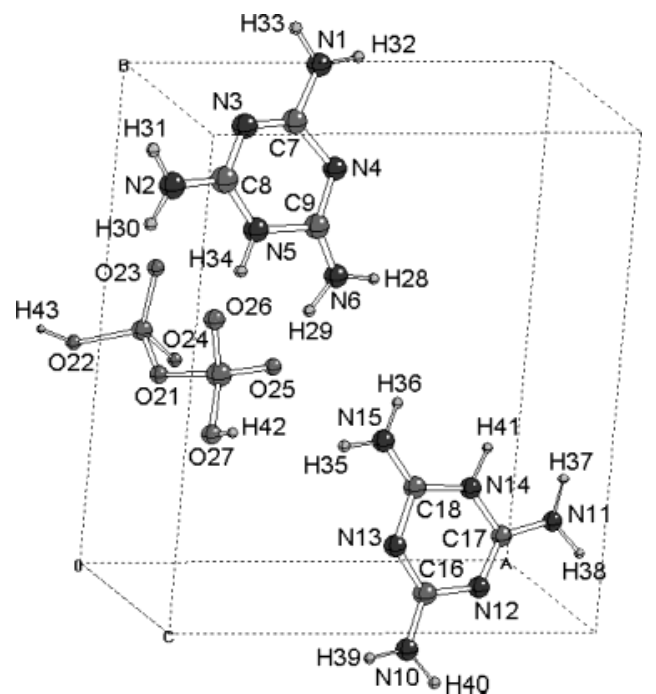

Figure 1. Labeling of the atoms in the asymmetric part of the melamine pyrophosphate unit cell.

are due to the steric effect of a lone pair of electrons, consistent with the valence-shell electron-pair repulsion theory. ${ }^{32}$

For completeness, it is mentioned that a refinement in space group $P 1$ has been carried out but this yielded neither significant structural changes nor an improvement of the $R$-factors.

Details of the structure refinement are listed in Table 1, and the refined atomic coordinates are given in Table 2. Observed and calculated diffraction patterns show good correspondence, even at high $2 \theta$ values (Figure 2).

Solid-State NMR Characterization. The hydrogen bonds and single protonation of the melamines as discussed above are corroborated by solid-state NMR data. Parts A and B of Figure 3 show a comparison of the single-pulse ${ }^{1} \mathrm{H}$ magic-anglespinning (MAS) solid-state NMR spectra of MP and MPy obtained at $800 \mathrm{MHz}$, using 49-kHz sample spinning to ensure spectral resolution.
Chemical shifts $>10$ ppm indicate protons involved in $\mathrm{R}-\mathrm{H} \cdot$ -O hydrogen bonding. ${ }^{33}$ Assignment of the ${ }^{1} \mathrm{H}$ chemical shifts in MP was done on the basis of ${ }^{1} \mathrm{H}-\mathrm{X}\left({ }^{31} \mathrm{P},{ }^{15} \mathrm{~N},{ }^{13} \mathrm{C}\right) \mathrm{HETCOR}$ experiments as is discussed more extensively elsewhere. ${ }^{15}$ In the MP spectrum the resonance at $13.7 \mathrm{ppm}$ stems from the $\mathrm{H}$ atom bonded to an endocyclic $\mathrm{N}$ atom, ${ }^{14,15}$ whereas the corresponding resonance $(13.1 \mathrm{ppm})$ in the MPy spectrum is only slightly shifted. In the MP spectrum the resonances at 11.1 and $13.2 \mathrm{ppm}$ result from the $\mathrm{H}$ atoms bonded to the phosphate oxygen atoms, while in the MPy spectrum, due to the condensation, only one of these resonances remains. This line is shifted considerably to $14.5 \mathrm{ppm}$, indicating a stronger hydrogenbonded network between the pyrophosphates in MPy in comparison with the orthophosphates in MP. ${ }^{33}$ The two peaks at 13.1 and $14.5 \mathrm{ppm}$ in the MPy spectrum appear in an intensity ratio of $1: 1$, establishing that the same number of $\mathrm{H}$ atoms is bonded to phosphate oxygen atoms as to endocyclic $\mathrm{N}$ atoms. The resonances of the exocyclic $\mathrm{NH}_{2}$ groups appear in both the MP and MPy spectrum in the chemical shift range 4-10 ppm, where the spectral differences support a change in the melamine stacking and bonding going from MP to MPy, although more detailed conclusions cannot be drawn. DFT calculations of proton chemical shifts could provide a deeper insight and are under investigation.

The phosphorus spectrum of MPy contains two resonances from apparently inequivalent phosphorus sites. The twodimensional ${ }^{1} \mathrm{H}-{ }^{31} \mathrm{P}$ heteronuclear correlation spectrum of MPy (Figure 3C) reveals that both ${ }^{31} \mathrm{P}$ resonances show strong crosspeaks with the ${ }^{1} \mathrm{H}$ resonance at $14.5 \mathrm{ppm}$, on the basis of which we can assign this proton resonance to two $\mathrm{H}$ atoms bonded to two phosphate oxygen atoms in the pyrophosphate. The crosspeaks to the ${ }^{1} \mathrm{H}$ resonances at 13.1 and $4-10 \mathrm{ppm}$ are much weaker, in line with their assignment to the endocyclic NH and exocyclic $\mathrm{NH}_{2}$ groups, respectively. Finally, Figure 4 shows the ${ }^{31} \mathrm{P}$ cross-polarization (CP) MAS spectrum of MPy at $4-\mathrm{kHz}$ sample-spinning frequency. A Herzfeld-Berger analysis ${ }^{34}$ of the sideband pattern for the two inequivalent phosphorus sites

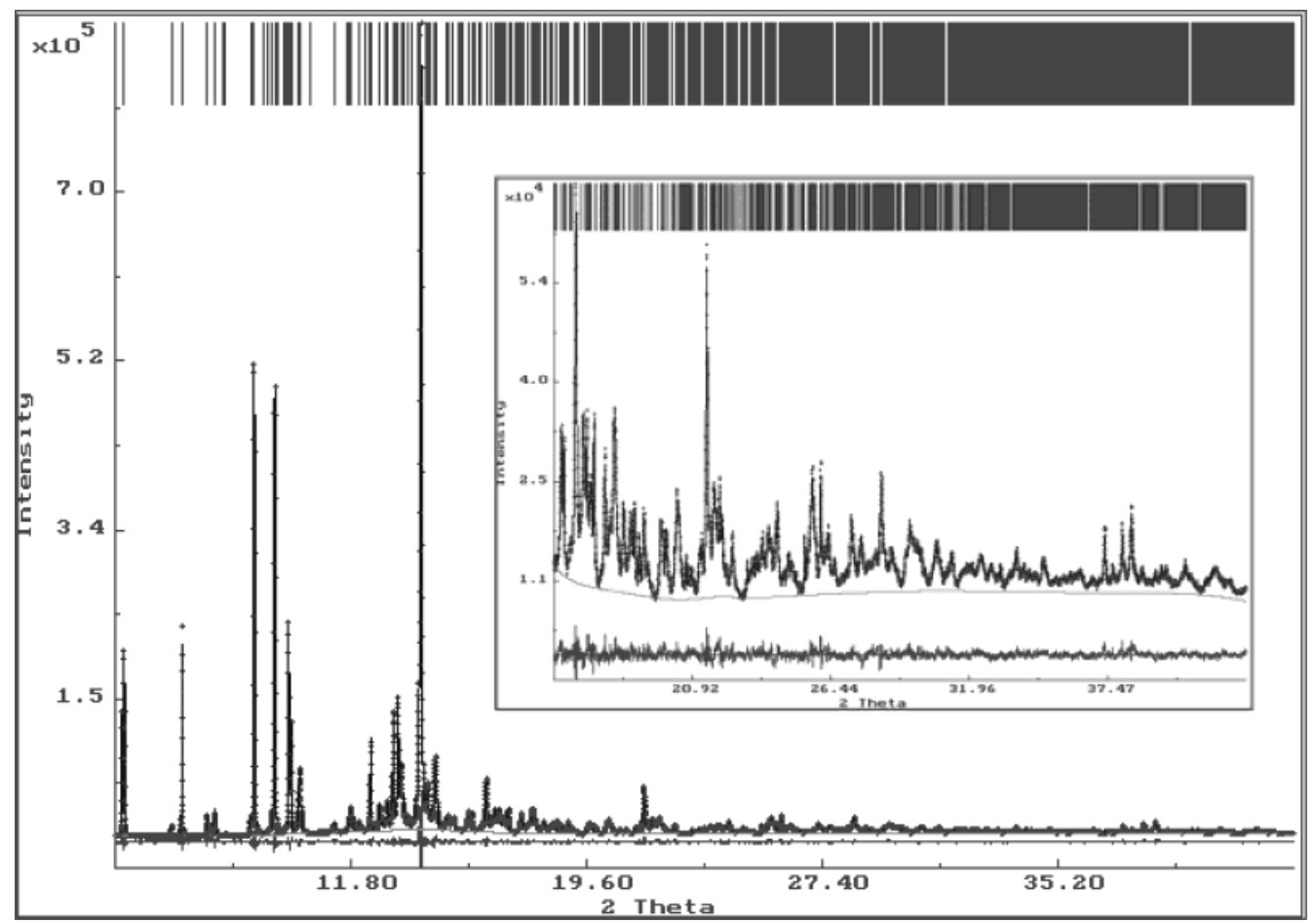

Figure 2. Synchrotron powder diffraction patterns of melamine pyrophosphate: experimental pattern (dots), the final calculated pattern after refinement (line), the calculated background and the difference (experimental - calculated) pattern. Reflection positions are marked at the top. 
TABLE 2: Atomic Coordinates and Displacement Factors (in $\AA^{2}$ ) for Melamine Pyrophosphate

\begin{tabular}{llllc}
\hline atom & \multicolumn{1}{c}{$x$} & \multicolumn{1}{c}{$y$} & \multicolumn{1}{c}{$z$} & $U_{\text {iso }}$ \\
\hline N1 & $0.7113(4)$ & $-0.1031(4)$ & $0.2520(7)$ & $0.0248(1)$ \\
N2 & $1.0277(4)$ & $0.1385(4)$ & $0.2560(7)$ & $0.0248(1)$ \\
N3 & $0.8688(4)$ & $0.0204(4)$ & $0.2614(8)$ & $0.0248(1)$ \\
N4 & $0.6521(4)$ & $0.1063(4)$ & $0.2472(7)$ & $0.0248(1)$ \\
N5 & $0.8202(4)$ & $0.2292(4)$ & $0.2627(8)$ & $0.0248(1)$ \\
N6 & $0.6251(4)$ & $0.3214(3)$ & $0.2596(7)$ & $0.0248(1)$ \\
C7 & $0.7527(5)$ & $0.0109(5)$ & $0.2595(10)$ & $0.0203(1)$ \\
C8 & $0.9062(5)$ & $0.1272(5)$ & $0.2558(9)$ & $0.0203(1)$ \\
C9 & $0.6846(5)$ & $0.2202(4)$ & $0.2402(10)$ & $0.0203(1)$ \\
N10 & $0.6185(4)$ & $-0.0687(4)$ & $0.7436(7)$ & $0.0248(1)$ \\
N11 & $0.9275(4)$ & $0.1882(4)$ & $0.7633(8)$ & $0.0248(1)$ \\
N12 & $0.7720(4)$ & $0.0539(4)$ & $0.7500(7)$ & $0.0248(1)$ \\
N13 & $0.5663(4)$ & $0.1403(4)$ & $0.7559(7)$ & $0.0248(1)$ \\
N14 & $0.7320(4)$ & $0.2538(4)$ & $0.7573(7)$ & $0.0248(1)$ \\
N15 & $0.5211(4)$ & $0.3474(4)$ & $0.7441(7)$ & $0.0248(1)$ \\
C16 & $0.6475(5)$ & $0.0427(5)$ & $0.7509(10)$ & $0.0203(1)$ \\
C17 & $0.8111(5)$ & $0.1576(5)$ & $0.7597(9)$ & $0.0203(1)$ \\
C18 & $0.6003(5)$ & $0.2580(5)$ & $0.7626(10)$ & $0.0203(1)$ \\
P19 & $0.14764(19)$ & $0.43144(19)$ & $0.7734(4)$ & $0.0383(1)$ \\
P20 & $0.24660(18)$ & $0.40223(18)$ & $1.1848(3)$ & $0.0383(1)$ \\
O21 & $0.1557(3)$ & $0.3709(3)$ & $0.9683(6)$ & $0.0380(1)$ \\
O22 & $0.0241(4)$ & $0.3862(3)$ & $0.6293(6)$ & $0.0380(1)$ \\
O23 & $0.1530(3)$ & $0.5666(4)$ & $0.8429(6)$ & $0.0380(1)$ \\
O24 & $0.2562(3)$ & $0.3569(3)$ & $0.6627(6)$ & $0.0380(1)$ \\
O25 & $0.3768(3)$ & $0.4117(3)$ & $1.1594(7)$ & $0.0380(1)$ \\
O26 & $0.1956(3)$ & $0.5299(3)$ & $1.3075(6)$ & $0.0380(1)$ \\
O27 & $0.2212(4)$ & $0.2948(4)$ & $1.2761(6)$ & $0.0380(1)$ \\
H28 & 0.766 & -0.16949 & 0.24852 & 0.0507 \\
H29 & 0.62971 & -0.11076 & 0.2506 & 0.0507 \\
H30 & 1.08325 & 0.07325 & 0.25995 & 0.0507 \\
H31 & 1.05358 & 0.21133 & 0.25218 & 0.0507 \\
H32 & 0.54583 & 0.32862 & 0.28273 & 0.0507 \\
H33 & 0.66097 & 0.38801 & 0.25062 & 0.0507 \\
H34 & 0.84662 & 0.31216 & 0.26392 & 0.0507 \\
H35 & 0.6782 & -0.13099 & 0.7385 & 0.0507 \\
H36 & 0.53882 & -0.08313 & 0.74367 & 0.0507 \\
H37 & 0.99126 & 0.12993 & 0.75845 & 0.0507 \\
H38 & 0.94176 & 0.26698 & 0.77056 & 0.0507 \\
H39 & 0.43853 & 0.33661 & 0.72564 & 0.0507 \\
H40 & 0.54732 & 0.42181 & 0.7494 & 0.0507 \\
H41 & 0.76452 & 0.33348 & 0.75514 & 0.0507 \\
H42 & 0.23808 & 0.32203 & 1.42958 & 0.0507 \\
H43 & -0.06244 & 0.41786 & 0.65333 & 0.0507
\end{tabular}

in MPy results in chemical shift anisotropies of $\left(\delta_{11}, \delta_{22}, \delta_{33}\right)$ $=(65,-8,-86) \pm 2 \mathrm{ppm}$ and $(67,-11,-91) \pm 2 \mathrm{ppm}$, which are in good agreement with literature data for dihydrogen pyrophosphate salts, further substantiating the assignments. ${ }^{35,36}$

The ${ }^{13} \mathrm{C}$ CP-MAS spectrum of MPy (not shown here) shows two resonances (2:1) identical with the ${ }^{13} \mathrm{C}$ spectrum of $\mathrm{MP},{ }^{14,15}$ leading to the conclusion that the carbon atoms in the melamine ring are inequivalent, in line with protonation of one endocyclic nitrogen per melamine, thus leaving the protonation scheme from MP to MPy unchanged.

Although the achieved ${ }^{1} \mathrm{H}$ spectral resolution is remarkable for powder samples containing a dense proton network, the relatively broad line width is attributed to residual dipolar couplings. ${ }^{11}$ As a result we cannot draw conclusions about the presence of chemical shift dispersions caused by local disorder or amorphous fractions on the basis of the proton spectra. In contrast, the ${ }^{13} \mathrm{C}$ and ${ }^{31} \mathrm{P}$ MAS spectra are well defined with relatively narrow lines. From this we conclude that the samples are highly crystalline and do not contain large quantities of amorphous fractions as is corroborated by the $\mathrm{X}$-ray diffraction results. The ${ }^{31} \mathrm{P}$ NMR spectra, however, do show the presence of impurities in the samples.

Theoretical Calculations. Additional support for the model of MPy presented here is also provided by quantum mechanical

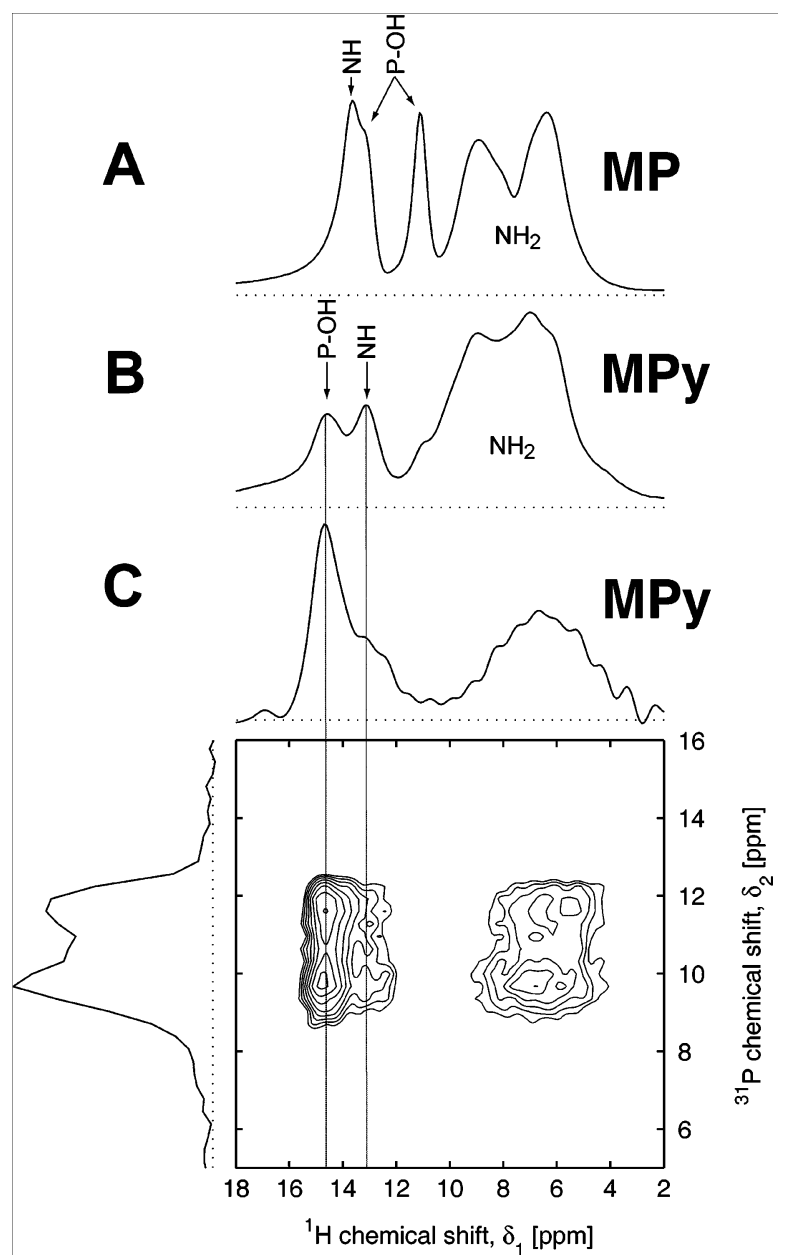

Figure 3. (A) Single-pulse ${ }^{1} \mathrm{H}$ MAS solid-state NMR spectrum of MP. (B) Corresponding spectrum of MPy. (C) Two-dimensional ${ }^{1} \mathrm{H}-{ }^{31} \mathrm{P}$ heteronuclear correlation spectrum of MPy. The $\delta_{2}$-axis represents the direct ${ }^{31} \mathrm{P}$ dimension, whereas the $\delta_{1}$-axis represents the indirect ${ }^{1} \mathrm{H}$ dimension. Cross-peaks indicate close through-space contacts between the corresponding nuclei.

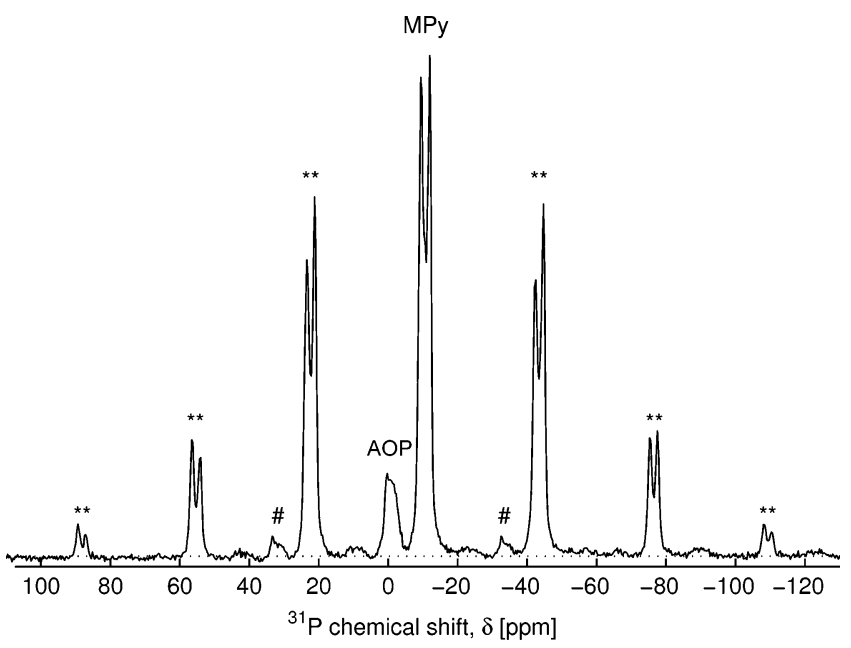

Figure 4. ${ }^{31} \mathrm{P}$ CP-MAS spectrum of MPy. The centerband and sidebands of the two inequivalent phosphorus sites in MPy are labeled (MPy) and (**), respectively. In addition the sample contains a fraction of an amorphous orthophosphate compound, for which the centerband and sidebands are labeled (AOP) and (\#), respectively.

periodic DFT calculations. After optimization of the MPy geometry with various positions of hydrogen atoms, all models had both melamine moieties single protonated. The DFT 


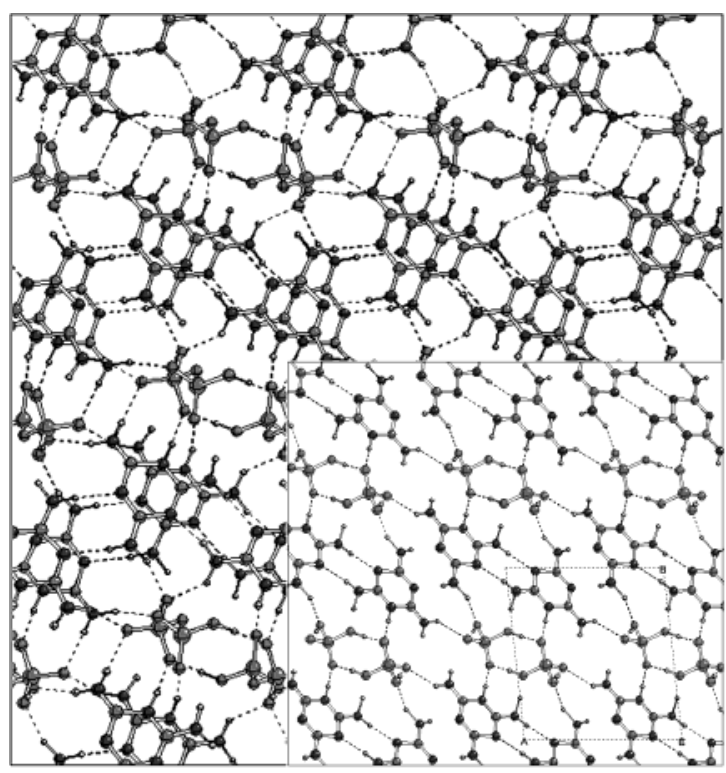

(A)

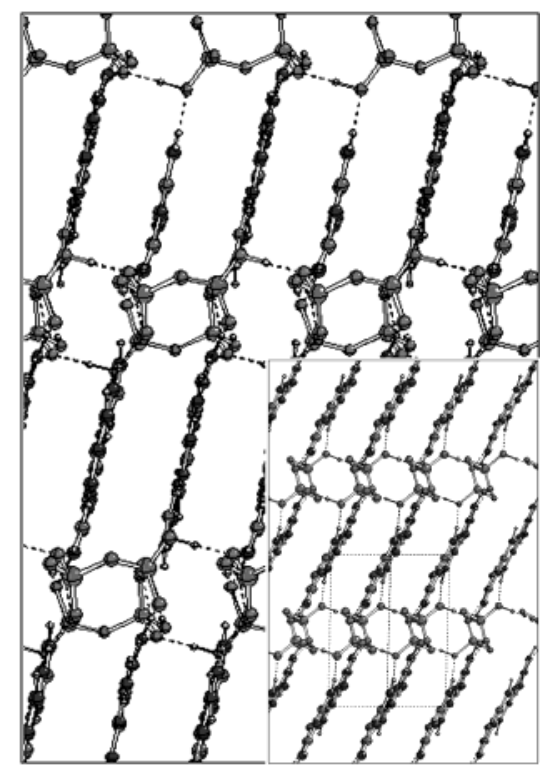

(B)

Figure 5. Crystal packing of MPy with the $c$ axis perpendicular to the plane of the paper (A) and with the $a$ axis perpendicular to the plane of the paper (B) and their corresponding views of MP shown in the insets.

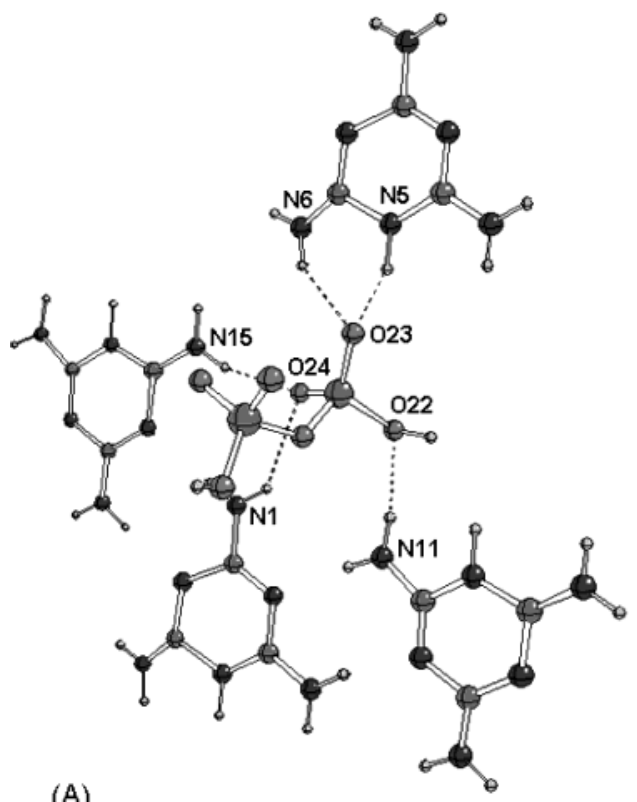

(A)

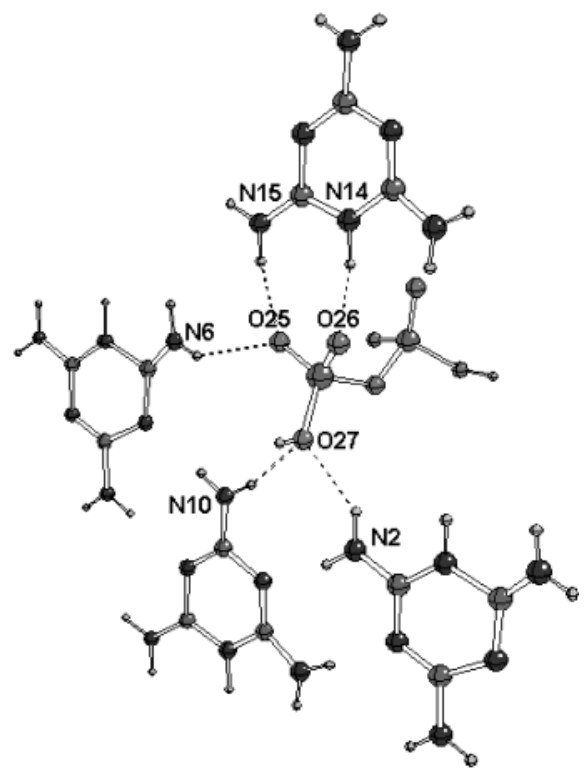

(B)

Figure 6. Hydrogen bonds between melamines and the two phosphate groups of the pyrophosphate moiety in MPy.

calculations did not allow the position of hydrogen atoms at the pyrophosphate moieties (energy differences of only 1.09 $\mathrm{kcal} / \mathrm{mol}$ ) to be identified uniquely. Still, the lowest total energy was found for the MPy model proposed here. The optimized structure is also in very good agreement with the X-ray model (RMS of only $0.15 \AA$ ).

\section{Discussion}

MPy Crystal Structure. The crystal structure of MPy consists of infinite chains of melamine cations and pyrophosphate anions with all molecules taking part in a vast hydrogenbonded network (Figure 5). Within the cation chains adjacent melamine moieties are linked by means of side-by-side pairs of $\mathrm{N}-\mathrm{H} \cdots \mathrm{N}$ hydrogen bonds $(\mathrm{N} \cdots \mathrm{N} \approx 2.90-3.09 \AA)$ forming infinite chains (Figure $8 \mathrm{~A}$ ) with all molecules lying in one plane.
The chains are parallel to the $a b$ plane and stacked along the $c$ axis forming parallel melamine sheets with an interlayer distance of $3.25 \AA$, which is shorter than the usual distance between $\pi$-aromatic ring systems $(\sim 3.4 \AA)^{37}$ and indicates $\pi-\pi$ interactions between the melamines. The melamine chains are crosslinked in the $b$ direction and coupled in the $c$ direction by pyrophosphate anions. The two phosphorus groups of the pyrophosphate anion are bonded to melaminium cations in different ways, as confirmed by NMR results (see above). For instance, oxygen $\mathrm{O} 23$ is at a hydrogen-bonding distance from the endocyclic nitrogen N5, and also has a weaker bond to the exocyclic N6 of the same melamine moiety (Figure 6A). The oxygen $\mathrm{O} 26$ in the other phosphorus group (Figure $6 \mathrm{~B}$ ) is hydrogen bonded to the endocyclic N14 but it has no significant hydrogen bonds to exocyclic nitrogens. Instead, the oxygen $\mathrm{O} 25$ 


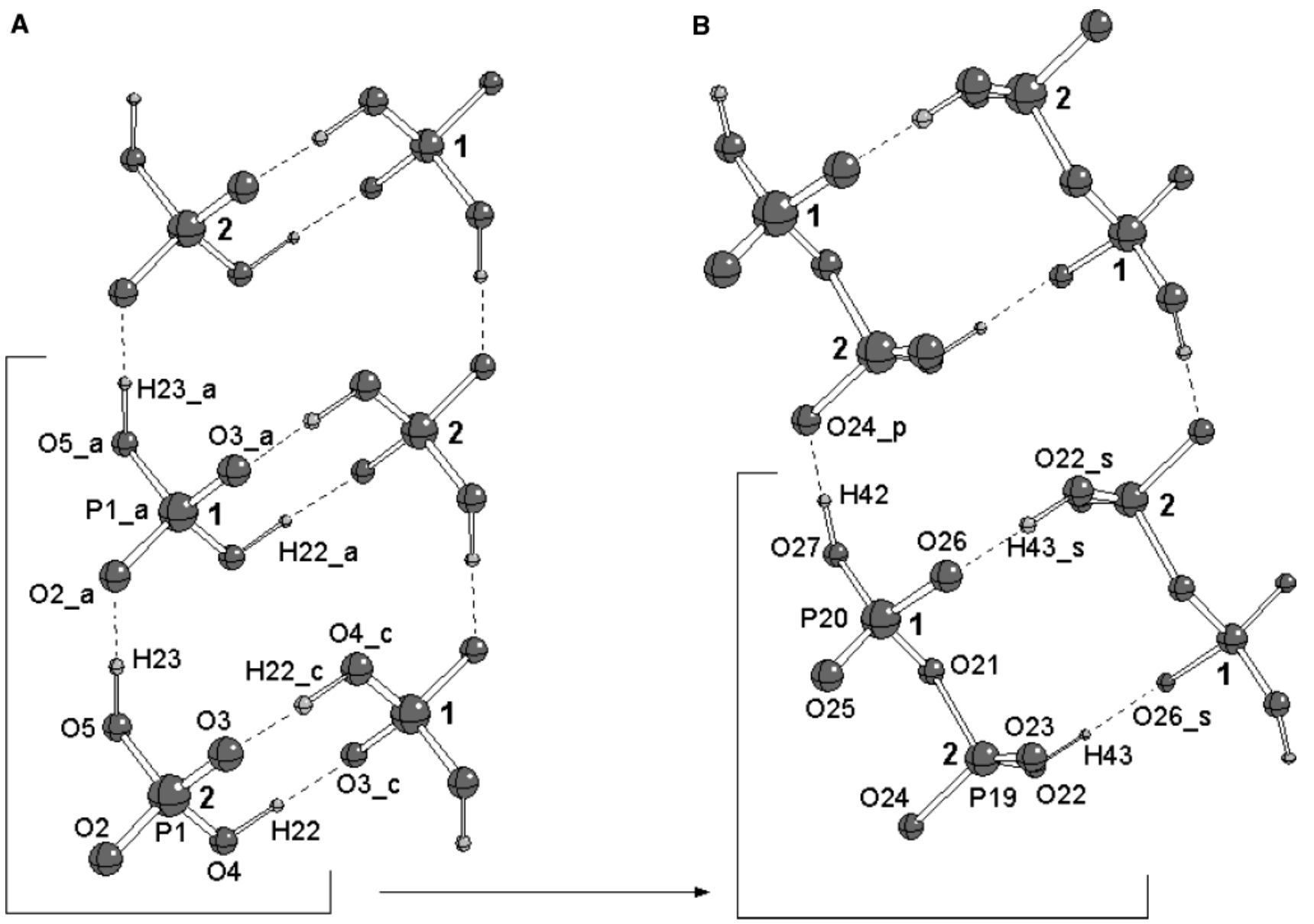

Figure 7. Pairs of phosphate chains in MP (A) and pyrophosphate chains in MPy (B). In the reaction from MP to MPy, the fragments marked by the solid line in part A become those marked in part B. The conformation of the phosphate groups marked "1" hardly changes in the reaction MP $\rightarrow$ MPy. In part A the subscripts_a, _c, and _ca correspond to the symmetry operations $x, y, 1+z,-x, 1-y, 1-z$, and $-x, 1-y, 2-z$, respectively, and in part B_p and _s correspond to $x, y, 1+z$ and $-x, 1-y, 2-z$, respectively.

(A)

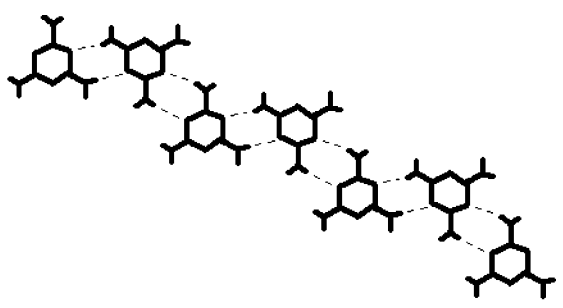

(B)

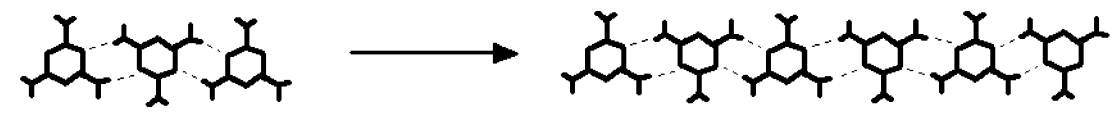

(C)

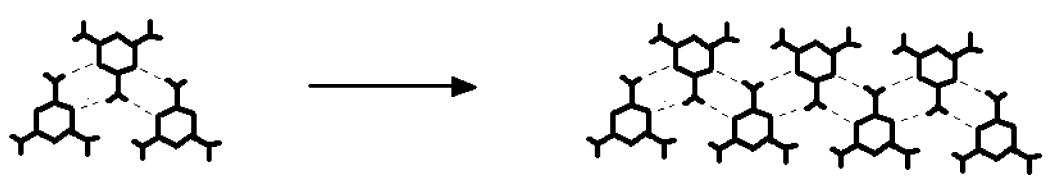

Figure 8. Three possible variants of the double hydrogen bonding system between melamines. From these schemes infinite melamine ribbons can be formed.

of this phosphorus group forms a hydrogen bond with the exocyclic nitrogen N15 of the same melamine moiety.

Every pyrophosphate moiety in MPy participates in two hydrogen bonds with neighboring anions as donor and in two bonds as acceptor (Figure 7B). Through these interactions the pyrophosphates form pairs of infinite chains with donor- acceptor $\mathrm{O} \cdots \mathrm{O}$ distances $\sim 2.52$ and $\sim 2.55 \AA$ along and between the chains, respectively, indicating a quite strong hydrogenbonding system as confirmed by the high ${ }^{1} \mathrm{H}$ chemical shifts for these protons. In MPy, as in MP, each phosphate moiety participates in four hydrogen bonds with other phosphates. In MPy the bonds are slightly shorter, indicating stronger interac- 
tion between pyrophosphates and being consistent with the increased ${ }^{1} \mathrm{H}$ chemical shifts of the $\mathrm{H}$ atoms bonded to the phosphate oxygen atoms (see above).

From MP to MPy. Both compounds consist of melamine sheets cross-linked in a similar manner by pairs of (pyro)phosphate chains, revealing that the condensation reaction proceeds along the chains, while in principle this reaction could proceed in either an intrachain or an interchain manner, see Figure 7.

Within the sheets, melamine molecules in MP and MPy bind in the same way, forming similar infinite chains (Figure 8A). However, the stacking of melamine sheets in these compounds is different. While in MPy all melamines in a sheet lie in the same plane, in MP pairs of neighboring melamine molecules do not lie in the same plane, but in parallel planes at close distance $(0.79 \AA)$ and being slightly tilted with respect to the plane of the sheet. The stacking distance between melamines from neighboring sheets in MPy $(\sim 3.25 \AA)$ is significantly smaller than that in MP $(\sim 3.42 \AA)$. The orientation of melamine sheets with respect to the (pyro)phosphate chain direction is also slightly different in MP and MPy.

Thus, in the reaction from MP to MPy the melamine sheets reorient with respect to (pyro)phosphate chains, resulting in a molecular arrangement with all the melamines in a sheet lying in the same plane and with a decreased stacking distance between melamines in neighboring sheets.

The bonding of one of the phosphorus groups of pyrophosphate in MPy to the melamines (Figure 6B) is similar to that of one of the phosphates in MP, i.e., half of the phosphates in MP more or less retained their conformation in MPy. These orthophosphates in MP are marked as $\mathbf{1}$ in Figure 7A (corresponding phosphorus groups in MPy are marked in the same way). The other half of the phosphates in MP, marked by 2 in Figure 7A, seem to have undergone a considerable rearrangement, with one of the $\mathrm{P}-\mathrm{O}$ bonds being broken to form a $\mathrm{P}-\mathrm{O}-\mathrm{P}$ bridge, but from the crystal structural data the precise rearrangement mechanism cannot be established.

On the basis of differential scanning calorimetry measurements it was established that the condensation of MP leading to formation of MPy is a strongly endothermic reaction. Energy calculations (see details in the Experimental Section) confirmed that in the crystal environment of MPy a single pyrophosphate unit plus a free water molecule have much higher energy $(\sim 23.6$ $\mathrm{kcal} / \mathrm{mol}$ ) than two orthophosphates in the crystal of MP. Nevertheless, this energy is about a factor of 2 lower than that calculated for a condensation reaction in the gas phase $(\sim 45-$ $55 \mathrm{kcal} / \mathrm{mol}^{38,39}$ ), implying that the effect of Coulomb repulsion between negatively charged phosphorus groups of pyrophosphate dianion moieties ${ }^{38-40}$ is partially compensated by crystalline packing effects, for example, melamine-phosphate bonding.

Common Packing Characteristics of Melamine-Containing Compounds. The melamine packing in MPy is quite common for melamine complexes and salts. While in pure melamine ${ }^{31}$ each melamine molecule binds through two hydrogen bonds to three neighbors, leading to a complex threedimensional melamine network, in the presence of other moieties melamine shows a typical tendency to self-assemble via double hydrogen bonds $(\mathrm{N} \cdots \mathrm{N} \approx 3 \AA)$ into infinite chains or "ribbons", stacked parallel to each other because of $\pi-\pi$ stacking effects. ${ }^{7,14,41-50}$ In these ribbons each melamine molecule is bonded to two melamine neighbors only because one of melamine's endocyclic nitrogens is hydrogen bonded to one of the other structural moieties, and therefore not available for hydrogen bonding to another (third) melamine. A literature search revealed that almost all melamine ribbons consist of an infinite stacking of one of three triple-melamine motifs (A, B, or $\mathrm{C}$; Figure 8) only. The sole exception to date has a bonded $\mathrm{A}-\mathrm{B}$ as its stacking motif. ${ }^{48}$ Apparently, the three types of ribbons found are efficient packings in which the number of inequivalent melamine moieties in the unit cell is minimized, although within each ribbon type variations of the shape occur such as inclined neighboring melamine molecules, ${ }^{41}$ or pairs of neighboring melamines being parallel but shifted with respect to each other as in MP, ${ }^{9}$ or all molecules being parallel and lying in one plane as in MPy.

The melamine phosphates solved to date (MPy, $\mathrm{MP},{ }^{14} \mathrm{M}_{6} \mathrm{P}_{5^{-}}$ $\left.\left(\mathrm{H}_{2} \mathrm{O}\right)_{4}{ }^{7}\right)$ suggest that melamine ribbons are likely to be present in other melamine phosphate compounds that have a melamineto-phosphorus ratio $\geq 1$ because each melamine in a ribbon has still one endocyclic nitrogen available for bonding and can easily accommodate one (ortho)phosphate. Consequently, lowering the melamine level implies a reduction of the chance that melamines will be able to assemble in double-hydrogen-bonded ribbons because of the increased competition with other nonmelamine moieties. ${ }^{46}$ This knowledge of melamine ordering motifs is expected to be helpful for the structure prediction of melamine phosphates in general. ${ }^{8}$

\section{Summary}

In conclusion, we characterized the crystal structure of MPy and discussed the structural changes that occur during the dehydration reaction MP $\rightarrow$ MPy. Also, the typical melamine packing in melamine complexes and salts was discussed. Further research into higher temperature condensation products of MPy and into the kinetics of these condensation reactions is in progress.

Acknowledgment. The authors acknowledge the ESRF (Grenoble, France) for the opportunity to perform the synchrotron diffraction experiments and Dr. H. Emerich for his help at beamline BM01B (Swiss-Norwegian CRG). They also thank E.J. Sonneveld and Dr. M. M. Pop for their help in data collection and indexing, Dr. R. B. Helmholdt, Dr. D. J. A. De Ridder, Dr. V. M. Litvinov, and Dr. K. Goubitz for useful discussions, and DSM for measurements of density of MPy. This work was supported by DSM (Geleen, The Netherlands), Ciba Speciality Chemicals (Basel, Switzerland), and The Netherlands Foundation for Scientific Research (NWO).

Supporting Information Available: Crystallographic CIF file. This material is available free of charge via the Internet at http://pubs.acs.org. Cambridge Crystallographic Data Center as publication no. CCDC-227043 contains the supplementary crystallographic data for this paper (Cambridge Crystallographic Data Centre, 12, Union Road, Cambridge CB2 1EZ, UK; fax (+44) 1223-336-033; or deposit@ccdc.cam.ac.uk).

\section{References and Notes}

(1) Weil, E.; McSwigan, B. J. Coat. Technol. 1994, 839(66), 75-82. 37.

(2) Jahromi, Sh.; Gabriëlse, W.; Braam, A. Polymer 2003, 44, 25-

(3) Zhu, W. M.; Weil, E. D.; Mukhopadhyay, S. J. Appl. Polym. Sci 1996, 62 (13), 2267-2280.

(4) Kandola, B. K.; Horrocks, A. R.; Horrocks, S. Fire Mater. 2001, $25,153-160$.

(5) Cichy, B.; Luczkowska, D.; Nowak, M.; Wladyka-Przybylak, M J. Eng. Chem. Res. 2003, 42, 2897-2905.

(6) Camino, G.; Costa, L.; Dicortemiglia, M. P. L. Polym. Degrad. Stab. 1991, 33 (2), 131-154. 
(7) Janczak, J.; Perpetuo, G. J. Acta Crystallogr., Sect. C 2002, 58, o455- 0459 .

(8) Frazler, A. W.; Waerstad, K. R.; Kim, Y. K. J. Chem. Eng. Data 1988, 333, 518-523.

(9) Harris, K., D., M. Cryst. Growth Des. 2003, 3 (6), 887-895.

(10) Schnell, I.; Brown, S. P.; Low, H. Y.; Ishida, H.; Spiess, H. W. J. Am. Chem. Soc. 1998, 120 (45), 11784-11795.

(11) Brown, S. P.; Spiess, H. W. Chem. Rev. 2001, 101 (12), 41254155.

(12) Goward, G. R.; Schnell, I.; Brown, S. P.; Spiess, H. W.; Kim, H.D.; Ishida, H. Magn. Reson. Chem. 2001, 39 (S1), S5-S17.

(13) Goward, G. R.; Sebastiani, D.; Schnell, I.; Spiess, H. W.; Kim,

H.-D.; Ishida, H. J. Am. Chem. Soc. 2003, 125 (19), 5792-5800.

(14) De Ridder, D. J. A.; Goubitz, K.; Brodski, V.; Peschar, R.; Schenk, H. Helv. Chim. Acta 2004, accepted for publication.

(15) Brinkmann, A.; van Eck, E. R. H.; Kentgens, A. P. M. Manuscript in preparation.

(16) Roberts, B.; Parrish, W. International Tables for Crystallography MacGillavry, C. H., Rieck, G. D., Eds.; Kynoch Press: Birmingham, 1962 Vol. III, pp 73-88. 64

(17) Samoson, A. Encyclopedia of NMR. In Adv. NMR 2002, 9, 59-

(18) Bennett, A. E.; Rienstra, C. M.; Auger, M.; Lakshmi, K. V.; Griffin,

R. G. J. Chem. Phys. 1995, 103, 6951-6958.

(19) Bielecki, A.; Kolbert, A. C.; Levitt, M. H. Chem. Phys. Lett. 1989, $155,341-346$.

(20) Ramamoorthy, A.; Wu, C. H.; Opella, S. J. J. Magn. Reson. 1999, $140,131-140$.

(21) Segall, M. D.; Lindan, P. L. D.; Probert, M. J.; Pickard, C. J.; Hasnip, P. J.; Clark, S. J.; Payne, M. C. J. Phys.: Condens. Matter 2002 14 (11), 2717-2743.

(22) Perdew, J. P.; Burke, K.; Ernzerhof, M. Phys. Rev. Lett. 1996, 77, $3865-3868$.

(23) Vanderbilt, D. Phys. Rev. Sect. B 1990, 41, 7892-7895.

(24) Visser, J. W. J. Appl. Crystallogr. 1969, 2, 89-95.

(25) Brodski, V.; Peschar, R.; Schenk, H. J. Appl. Crystallogr. 2003, $36,239-243$.

(26) Adams, J. M.; Ramdas, V. Acta Crystallogr., Sect B 1977, 33 , $3654-3657$

(27) Zlokazov, V. B.; Chernyshev, V. V. J. Appl. Crystallogr. 1992 $25,447-451$

(28) Toraya, H. J. Appl. Crystallogr. 1986, 19, 440-447.

(29) Dollase, W. A. J. Appl. Crystallogr. 1986, 19, 267-272.

(30) Baerlocher, C. The Rietveld Method; Young, R. A., Ed.; Oxford University Press: Oxford, 1993; pp 187-188.
(31) Varghese, J. N.; O'Connell, A. M.; Maslen, E. N. Acta Crystallogr., Sect. B 1977, 33, 2102-2108.

(32) (a) Gillespie, R. J. J. Chem. Educ. 1963, 40, 295-301. (b) Gillespie, R. J. Chem. Soc. Rev. 1992, 21, 59-69.

(33) Harris, R. K.; Jackson, P.; Merwin, L. H.; Say, B. J.; Hagele, G. J. Chem. Soc., Faraday Trans. 1988, 84, 3649-3672.

(34) Herzfeld, J.; Berger, A. E. J. Chem. Phys. 1980, 73, 6021-6030.

(35) Duncan, T. M. A Compilation of Chemical Shift Anisotropies; Farragut Press: Chicago, 1990

(36) Hartmann, P.; Vogel, J.; Schnabel, B. J. Magn. Reson. A 1994, 111 (1), $110-114$.

(37) Pauling, L. The Nature of the Chemical Bond, 3rd ed.; Cornell University Press: Ithaca, 1960; p 262.

(38) Colvin, M. E.; Evleth, E.; Akacem, Y. J. Am. Chem. Soc. 1995 $117,4357-4362$.

(39) Ma, B.; Meredith, C.; Schaefer, H. F., III J. Phys. Chem. 1994, 98, $8216-8223$

(40) Wang, X.; Vorpagel, E. R.; Yang, X.; Wang, L. J. Phys. Chem. A 2001, 105, 10468-10474.

(41) Gordon, L. E.; Harrison, W. T. A. Acta Crystallogr., Sect. E 2003 , 59, o195-o197.

(42) (a) Janczak, J.; Perpetuo, G. J. Acta Crystallogr., Sect. C 2001, 57 , 1120-1122. (b) Janczak, J.; Perpetuo, G. J. Acta Crystallogr., Sect. C 2002, 58, o339-0341. (c) Janczak, J.; Perpetuo, G. J. Acta Crystallogr., Sect. C 2001, 57, 873-875. (d) Janczak, J.; Perpetuo, G. J. Acta Crystallogr., Sect. C 2002, 58, o112-o114. (e) Janczak, J.; Perpetuo, G. J. Acta Crystallogr., Sect. C 2001, 57, 123-125. (f) Janczak, J.; Perpetuo, G. J. Acta Crystallogr., Sect. C 2004, 60, o211-o214.

(43) Tanbug, R.; Kirschboum, K.; Pinkerton, A. A. J. Chem. Crystallogr 1999, 29, 45-55.

(44) Zhang, Y. G.; Li, J. M.; Nishiura, M.; Imamoto, T. Chem. Lett. 1999, 7, 543-544.

(45) Chowdhry, M. M.; Mingos, D. M. P.; White, A. J. P.; Williams D. J. Chem. Commun. 1996, 8, 899-901.

(46) Lange, R. F. M.; Beijer, F. H.; Sijbesma, R. P.; Hooft, R. W. W.; Kooijman, H.; Spek, A. L.; Kroon, J.; Meijer, E. W. Angew. Chem. 1997, 109, 1006-1008; Angew. Chem., Int. Ed. Engl. 1997, 36, 969-971.

(47) Scoponi., M.; Polo, E.; Pradella, F.; Bertolasi, V.; Carassiti, V. Goberti, P. J. Chem. Soc., Perkin Trans. 1992, 2, 1127-1132.

(48) Janczak, J.; Perpetuo, G. J. Acta Crystallogr., Sect. C 2001, 57, $1431-1433$

(49) Marchewka, M. K.; Pietraszko, A. J. Phys. Chem. Solids 2003, 64, 2169-2181.

(50) Jing, H.; Strobele, M.; Weisser, M.; Meyer, H.-J. Z. Anorg. Allg. Chem. 2003, 629, 368-370. 